\title{
Prevalence of pathological neck of femur fractures in patients undergoing arthroplasty at a tertiary referral hospital
}

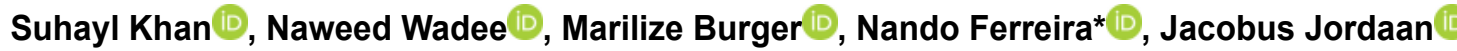 \\ Division of Orthopaedic Surgery, Department of Surgical Sciences, Faculty of Medicine and Health Sciences, Stellenbosch University, Cape Town, South Africa
}

${ }^{*}$ Corresponding author: nferreira@sun.ac.za

Citation: Khan S, Wadee N, Burger M, Ferreira N, Jordaan K. Prevalence of pathological neck of femur fractures in patients undergoing arthroplasty at a tertiary referral hospital. SA Orthop J 2021;20(1):16-20. http://dx.doi.org/10.17159/23098309/2021/v20n1a1

Editor: Dr David North, Paarl Hospital, Western Cape, South Africa

Received: August 2020

Accepted: October 2020

Published: March 2021

Copyright: () 2021 Khan S. This is an open-access article distributed under the terms of the Creative Commons Attribution Licence, which permits unrestricted use, distribution and reproduction in any medium, provided the original author and source are credited.

Funding: No funding was secured for this study.

Conflict of interest: The authors declare they have no conflicts of interest that are directly or indirectly related to the research.

\begin{abstract}
\section{Background}

This study aimed to determine the prevalence of pathological neck of femur (NOF) fractures at a tertiary referral hospital through histological examination of specimens in all NOF fracture patients undergoing hip arthroplasty. A secondary aim was to determine whether the current practice of sending all femoral heads for histological evaluation, to avoid missing unsuspected malignancies, is financially warranted.
\end{abstract}

\section{Methods}

A retrospective folder review of patients who underwent arthroplasty for NOF fractures was conducted. Patients with suspected pathological fractures were managed by the divisional Bone Tumour Unit while fragility traumatic fractures were managed by the Arthroplasty Unit. All femoral heads were sent for histological analysis regardless of suspicion of pathological fracture. Quotes from the public and private sector were sought to determine cost implications of sending femoral head specimens for histology

\section{Results}

A total of 311 patients were included. Of these, 11 patients (4\%) had suspected pathological fractures, with fragility/traumatic fractures being diagnosed in the remaining 300 patients $(97 \%)$. Histology results were available for 195 patients (63\%), including all the patients with suspected pathological fractures. No unexpected malignant histological results were observed, while nine of the suspected pathological fracture group had pathological fractures, confirmed with histology.

\section{Conclusion}

Pathological lesions were identified in $3 \%$ of patients undergoing arthroplasty for NOF fractures in our population, which is higher than other reports in the literature. Routine histological screening of femoral heads to exclude pathological fracture might not be necessary and cost effective, as pathological lesions can accurately be identified by clinical and radiographic evaluation.

\section{Level of evidence: Level 4}

Keywords: femoral head histology, pathological fracture, neck of femur fracture, metastases, hip arthroplasty

\section{Introduction}

Femoral neck fractures pose a significant burden to the healthcare system in developed and developing countries, with the annual incidence expected to increase in the coming years. ${ }^{1}$ The reason for this increase is suggested to be decreasing global mortality rates and the ageing populations' subsequent risk for osteoporosis. ${ }^{2}$ Over $84 \%$ of elderly patients with femoral neck fractures are reported to have underlying osteoporosis. ${ }^{3}$ Osteoporotic or fragility femoral neck fractures result in significant morbidity and mortality. Most patients never achieve pre-fracture functional status after surgical intervention, ${ }^{4}$ and up to $33 \%$ die within the first 12 months post-operatively. ${ }^{5}$

A small minority of patients sustain femoral neck fractures because of underlying pathological lesions. ${ }^{6}$ Pathological fractures may be caused by any bone lesion (benign, primary malignant or metastatic), but metastatic bone tumours and multiple myeloma are far more prevalent than other primary bony malignancies in the elderly population. ${ }^{7}$ The American Cancer Association expects around 1.8 million new cancer cases to be diagnosed in $2020 .^{8}$ There is also a steady decrease in mortality rate of $1.5 \%$ per year in patients with cancer and, as the population's life expectancy increases, there is an increased prevalence of bony metastases with subsequent risk of pathological fractures. ${ }^{9}$

Breast, thyroid, kidney, lung and prostate primary malignancies have a predilection for bony metastases, though any primary malignancy can metastasise to bone. ${ }^{10}$ The vertebral column is most commonly affected by bony metastases while the proximal femur is the most common site for metastases to the appendicular skeleton. ${ }^{11}$ This, along with the strong deforming forces across 
the hip joint, disproportionately predisposes the proximal femur to pathological fractures. Fifty per cent of pathological fractures occur at the femoral neck, $20 \%$ at the intertrochanteric area, and the remaining $30 \%$ in the rest of the femur. ${ }^{11}$

There are two studies in the English literature documenting the prevalence of pathological neck of femur (NOF) fractures. Ramisetty et al., in their review of 2223 consecutive NOF fractures, conducted in Birmingham, England, reported ten patients $(0.004 \%)$ with pathological fractures. ${ }^{6}$ This figure, however, does not represent true prevalence as only 90 of the 2223 patients in their cohort had histological evaluation. Davis et al. in their retrospective review at a level 1 trauma centre in California, United States of America, evaluated 850 consecutive femoral neck fracture patients, and found no unexpected malignancy in any of the 466 (54.8\%) specimens which were sent for histological evaluation. ${ }^{12}$ Similarly, true prevalence of pathological fractures in this study cannot be deduced as $45.2 \%$ of femoral heads were not sent for histological analysis, and hence missed pathological fractures cannot be definitively excluded.

True incidence of pathological femoral neck lesions in patients undergoing elective arthroplasty of the hip, in stark contrast, has been extensively researched. ${ }^{13-16}$ However, there is no clear consensus on whether it is necessary from an economic standpoint to send all resected specimens for histological examination in these patients. ${ }^{15,16}$

The aim of this study was to determine the prevalence of pathological NOF fractures at a tertiary referral hospital between 2014 and 2016 through histological specimens in all NOF fracture patients presenting for hip arthroplasty. The secondary aim was to determine if the current practice of sending all femoral heads for histological evaluation, to avoid missing unsuspected malignancies, is warranted, and to explore the financial cost involved.

\section{Methods}

A retrospective, observational review of case notes, imaging studies and histological results of all patients who underwent arthroplasty for NOF fractures between January 2014 and December 2016 was conducted.

As per institutional protocol, on admission all patients were divided into two groups depending on suspicion of pathological or fragility/ traumatic NOF fractures according to clinical and radiographic findings. Patients with suspected pathological fractures were subsequently managed by the divisional Bone Tumour Unit while fragility and traumatic NOF fractures were managed by the Arthroplasty Unit (Figure 1). Clinical features raising concern of possible pathological fractures included: i) antecedent hip pain, ii) atraumatic mechanism of injury and iii) a history of malignancy. Radiological features suggestive of pathological fractures included atypical fracture patterns (e.g. transverse fractures with minimal trauma) or any bony lesions (e.g. lytic, permeative, moth-eaten lesions) at the fracture site or any other bone on the radiograph.

Patients were identified from records from both units. All patients that underwent a head-sacrificing procedure were included in the study while patients who received arthroplasty for reasons other than NOF fractures and patients treated with internal fixation were excluded. There was no exclusion for age.

All resected femoral heads were sent for histology regardless if malignancy was suspected or not (Figure 1) as per our current standard of care for these fractures. For the fragility/traumatic fracture group, the type of hip reconstruction following head resection was selected on an individualised approach but was based on the National Institute of Health and Care Excellence (NICE) guidelines. Cognitively sound community ambulators who are anaesthetically medically fit are offered total hip arthroplasty over hemiarthroplasty. ${ }^{17}$

Patients who presented with a suspicious fracture without a known primary malignant lesion were investigated in order to find the primary lesion and exclude a primary malignancy of bone and were then offered surgery. Patients with known metastasis (and those where the primary malignant lesion causing metastasis was found) were offered bipolar hemiarthroplasty with long cemented stems to prevent periprosthetic fracture. Primary implants used for fragility/traumatic group fractures were the DePuy Johnson \& Johnson Corail Pinnacle system. Primary implants used for

Patients presenting with femur neck fracture

Clinical evaluation

- History of malignancy or metastases

- Constitutional symptoms

- Atraumatic mechanism of injury

- Antecedent hip pain

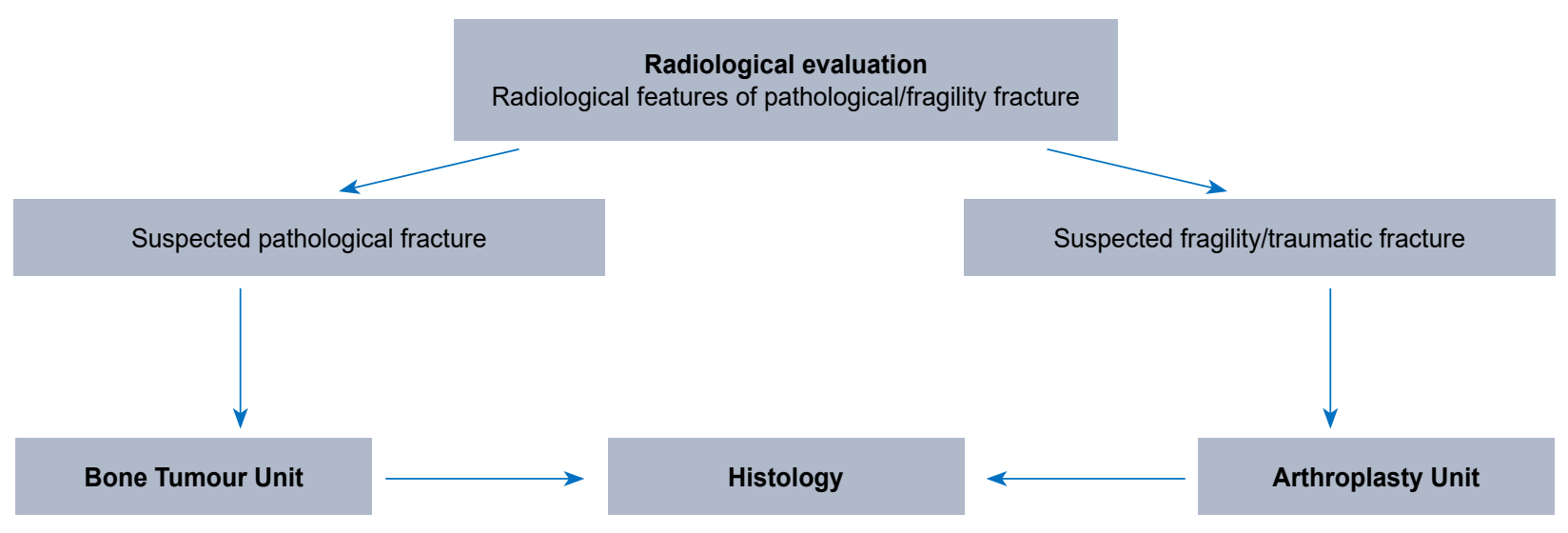

Figure 1. Flow diagram of patients presenting with neck of femur fractures 
pathological fractures were Stryker Exeter V40 long femoral stems with bipolar hemi-arthroplasty heads.

To determine the financial implications of sending femoral heads for pathological examination, The National Laboratory Health Service, which provides histopathological examination for specimens in the state sector, as well as Pathcare, which services the private sector, provided quotes in November 2019 of routine processing of these specimens.

Data was analysed using STATISTICA v13. Continuous demographic data (age) is reported as a mean and standard deviation together with $95 \%$ confidence intervals $(\mathrm{Cl})$. Categorical data is described as frequencies and/or counts.

\section{Results}

A total of 311 patients who underwent arthroplasty for NOF fractures were included in this study. The cohort comprised 215 women $(69 \%)$ and 96 men (31\%) with a mean age of 73.4 years $\pm 12.6(95 \% \mathrm{Cl} 72-74$.).

A total of 11 of 311 patients (4\%) were admitted with suspected pathological fractures, based on clinical and/or radiological suspicion with fragility/traumatic fractures being diagnosed in the remaining 300 patients (97\%). Histology results were available for 195 patients $(63 \%)$ including all of the patients with suspected pathological fractures, and 184 of the suspected traumatic/fragility fractures.

Nine of the suspected 11 patients were histologically confirmed to have a malignancy (Table I), all of which were secondary to metastatic disease or multiple myeloma, with no primary sarcomas diagnosed. Of these, four out of nine (44\%) patients presented with an unknown primary malignancy and required a thorough clinical examination and basic haematological (tumour markers, myeloma workup) and radiological (CT chest/abdomen/pelvis) investigations to identify the primary malignancy prior to surgery. The histology from the resected femoral specimens for these patients confirmed the respective primary malignancy after initial workup.

Breast and lung carcinoma were the most common source of the primary malignancies, accounting for six of the nine pathological fractures (three each). Multiple myeloma, renal and prostate carcinoma accounted for the other three patients (Table I, Figure 2).

The first of the two suspected pathological fracture patients who did not have a pathological lesion on histology was a 68-yearold male known with prostate cancer and who had a suspicious femoral neck lytic lesion on radiographs, not in keeping with the blastic metastasis usually associated with prostate cancer. After MRI (hip), CT (chest, abdomen and pelvis) and bone scan were non-contributory, the lytic lesion was biopsied to exclude a primary bony sarcoma or second primary malignancy. The biopsy showed no signs of pathological fracture and he subsequently received a total hip replacement. Histological evaluation of the resected femoral head confirmed the absence of any pathological lesions.

The second patient was a 47-year-old HIV-positive female with a large breast mass with skin involvement. This was her index presentation to any healthcare facility for the breast mass. Radiographs revealed a subcapital NOF fracture and suspicious per trochanteric lytic lesion. She was assumed to have a pathological fracture secondary to metastasis from breast cancer and hence received a long-cemented stem bipolar hemiarthroplasty. Biopsy of the breast mass was done concurrently, and she was subsequently discharged to the care of the breast surgical oncology unit for further management. The breast biopsy confirmed malignancy (invasive carcinoma); however, histology of the femoral head did not show any pathology.

Three of the nine (33\%) patients with pathological fractures presented with antecedent hip pain, five (56\%) with a known primary malignancy, three (33\%) with known metastatic disease, and two $(22 \%)$ with an atraumatic mechanism of injury. All nine patients had radiographic features of pathological fractures (Figure 3).

Histological results were available for 184 (of 300) patients in the fragility/trauma group. There were no unexpected malignant histological results found in this group. In 116 patients no

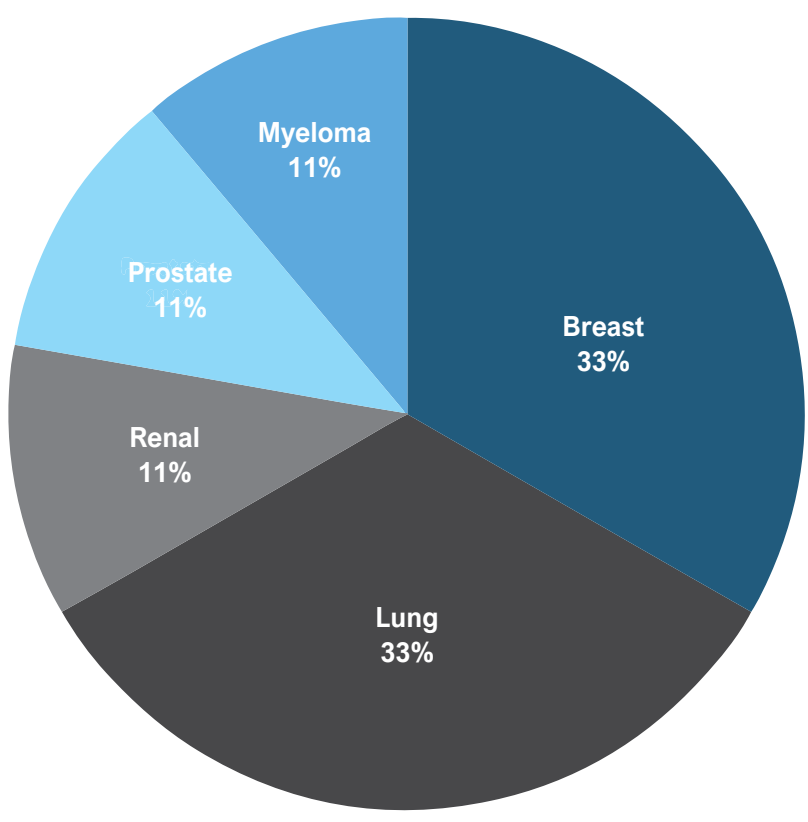

Figure 2. Percentage of primary malignancies responsible for metastatic lesions

Table I: Patients with pathological findings on histology

\begin{tabular}{|c|c|c|c|c|c|c|c|}
\hline $\begin{array}{c}\text { Age } \\
\text { (years) }\end{array}$ & Sex & $\begin{array}{l}\text { Known } \\
\text { primary }\end{array}$ & $\begin{array}{c}\text { Known } \\
\text { metastases }\end{array}$ & $\begin{array}{l}\text { X-ray features of } \\
\text { malignancy }\end{array}$ & $\begin{array}{c}\text { Atraumatic } \\
\text { mechanism of injury }\end{array}$ & $\begin{array}{l}\text { Antecedent } \\
\text { hip pain }\end{array}$ & Histology results \\
\hline 65 & Female & No & No & Lytic & No & No & Metastatic lung carcinoma \\
\hline 67 & Female & No & No & Sclerotic & Yes & No & Metastatic lung carcinoma \\
\hline 75 & Female & Yes & Yes & Permeative & No & No & Metastatic breast carcinoma \\
\hline 54 & Female & Yes & Yes & Lytic & No & Yes & Metastatic breast carcinoma \\
\hline 51 & Female & No & No & Permeative & No & Yes & Metastatic lung carcinoma \\
\hline 87 & Male & Yes & No & Sclerotic & No & No & Metastatic prostate carcinoma \\
\hline 54 & Female & No & No & Lytic & No & No & Multiple myeloma \\
\hline 72 & Female & Yes & Yes & Lytic & No & No & Metastatic renal carcinoma \\
\hline 54 & Female & Yes & No & Lytic & Yes & Yes & Metastatic breast carcinoma \\
\hline
\end{tabular}




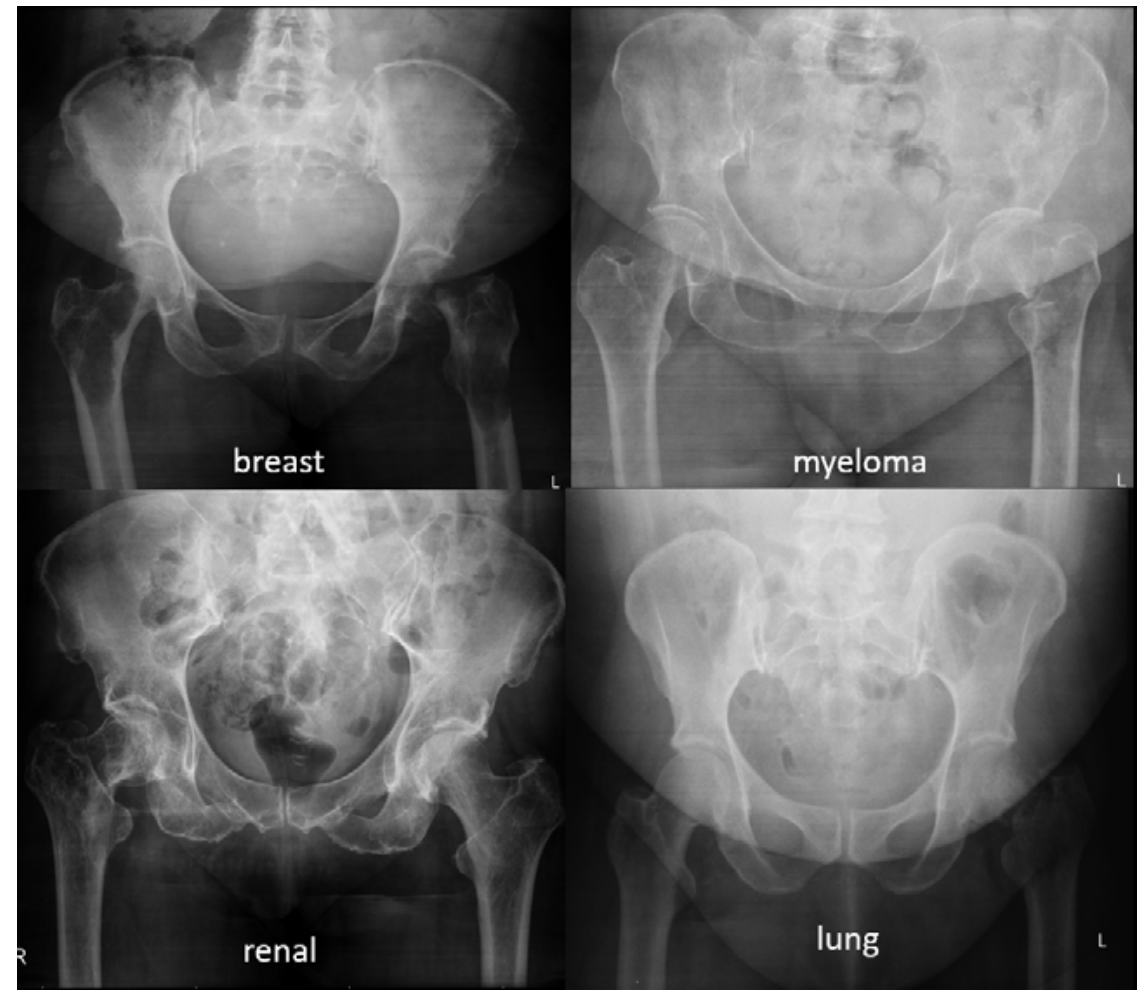

Figure 3. Anteroposterior radiographs of patients from our study presenting with pathological fractures with the source of the primary malignancy denoted below each image

histological results could be found. The reason these 116 excised femoral heads were not histologically analysed as per institutional protocol was difficult to define retrospectively. Lost specimens, problems with transport of specimens from theatre to the pathology lab, human error, resulting in the specimens not being ordered are all considerations.

All but one of the patients with pathological fractures were treated with a long-cemented stem bipolar hemiarthroplasty. This patient returned two weeks post-surgery with a periprosthetic fracture below the implant that required revision. There was no major intraoperative or immediate post-operative morbidity or mortality related to cementation in patients who received long cemented stems for pathological fractures.

The approximate cost of a single femoral head histological examination in the private healthcare sector and the state healthcare system was ZAR 1956 (US\$ 134) and ZAR 540 (US\$ 37) respectively.

\section{Discussion}

The aim of the study was to establish the prevalence of pathological fractures at our institution and to determine the financial implications of sending all femoral heads for histological examination.

The first main finding of this study was that all pathological femoral neck fractures were secondary to metastatic disease and represented $3 \%$ of patients in the total cohort ( 9 of 311 ), or $5 \%$ (9 of $195)$ of those with confirmed histology findings. This is considerably higher than that reported by Davis et al. $(0.9 \%)^{12}$ and Ramisetty et al. $(0.45 \%) .{ }^{6}$ No conclusive reason can be given for this disparity in prevalence without further comparative studies. It could be explained by the lower number of femoral heads sent for histological examination (52\% and $4 \%$ respectively), perhaps resulting in missed pathological fractures. More likely, it represents the delayed presentation due to health-seeking behaviour ${ }^{18}$ and lack of comprehensive screening programmes for cancer; ${ }^{19}$ hence the delay in treatment, increasing the prevalence of metastatic disease in the South African context. The patients with pathological fractures presented at a younger age (mean 63 years) than those with fragility fractures (mean 73 years), and this should be considered when treating these patients.

In view of the fact that there were no missed pathological fractures, and that nine of 11 patients with pathological lesions were correctly identified on clinical features and radiographic findings in our series, sending all femoral heads routinely seems unnecessary. Almost half of the pathological fractures in the current series presented without a known primary malignancy, and in all these cases the primary was preliminarily identified using Rougraff et al.'s ${ }^{20}$ rudimentary diagnostic approach comprising basic blood tests and a computed tomography scan of the chest, abdomen and pelvis. Three of the four unknown primary tumours were of lung origin, in keeping with the nature of bronchogenic malignancies as they have few clinical symptoms initially and metastasise early, with metastatic lesions often the first manifestation of the disease. ${ }^{21}$ There were no pathological fractures due to bony sarcomas consistent with the rarity of primary malignant bony lesions in elderly patients. ${ }^{22}$ All four patients who presented with metastases of an 'unknown primary' had a first histological diagnosis made on their femoral head specimens, as this preceded biopsy from the area of primary malignancy. This highlights the importance of pathological examination of the femoral heads from suspected lesions.

There is controversy in the literature regarding the use of short uncemented or longer cemented stems for patients with pathological fractures. Short uncemented stems weigh the risk of prosthetic loosening or periprosthetic fracture in the future, with the immediate risk of intra-operative haemodynamic instability or death with bone cement implantation syndrome associated with longer cemented stems. ${ }^{11,22}$ In the current series we did not experience any cementation-related complications in patients who received long cemented stems for pathological fractures.

A secondary aim was to determine the cost implications of sending all femoral heads for histological evaluation. In this study, femoral head histology $(n=195)$ amounted to between ZAR 105300 (US\$ 7 274) and ZAR 381420 (US\$ 26 322) over the three-year period. As we were able to accurately exclude malignancy using clinical and radiographic criteria, sending only the suspicious femoral heads $(n=11)$ for histological examination would have resulted in a reduced cost of between ZAR 99360 (US\$ 6 864) and ZAR 359904 (US\$ 24 838). As femoral neck fracture incidence is increasing globally, ${ }^{1}$ and considering financial constraints on the healthcare system, judicious use of resources is imperative. Subsequently, the findings of this study confirm the recommendation that clinicians should consider sending only the suspected pathological fractures for histological evaluation.

The limitations of this study include its retrospective nature and the fact that not all femoral heads were sent for pathological examination. We are therefore not able to definitively exclude pathological fractures for all patients, although we report a higher percentage than previous studies. 


\section{Conclusion}

Pathological lesions were identified only in a small percentage of patients undergoing arthroplasty for NOF fractures in our population, albeit higher than other studies in the literature. Routine histological screening of femoral heads to exclude pathological fracture might not be necessary, as pathological lesions can accurately be identified by clinical and radiographic evaluation.

\section{Ethics statement}

The authors declare that this submission is in accordance with the principles laid down by the Responsible Research Publication Position Statements as developed at the 2nd World Conference on Research Integrity in Singapore, 2010.

Prior to commencement of the study ethical approval was obtained from the following ethical review board: Health Research Ethics Committee, Stellenbosch University, Project ID 8744 and ethics reference number S18/10/264. All procedures were in accordance with the ethical standards of the responsible committee on human experimentation (institutional and national) and with the Helsinki Declaration of 1975 as revised in 2008. Informed written consent was not obtained from all patients for being included in the study.

\section{Declaration}

The authors declare authorship of this article and that they have followed sound scientific research practice. This research is original and does not transgress plagiarism policies.

\section{Author contributions}

SK: Data capture, manuscript preparation

NW: Data capture, manuscript preparation

MB: Data analysis, manuscript revision

NF: Study conceptualisation, data capturing, study design, manuscript revision

$\mathrm{KJ}$ : Study conceptualisation, data capturing, study design, manuscript revision

\section{References}

1. Johnell O, Kanis J. Epidemiology of osteoporotic fractures. Osteoporos Int. 2005;16(suppl. 2):6-10. https://doi.org/10.1007/s00198-004-1702-6.

2. Thorngren KG, Hommel A, Norrman PO, Thorngren J, Wingstrand $\mathrm{H}$. Epidemiology of femoral neck fractures. Injury. 2002;33(suppl 3):C1-7. http:// www.ncbi.nlm.nih.gov/pubmed/12423584.

3. Cummings SR, Browner W, Cummings SR, et al. Bone density at various sites for prediction of hip fractures. Lancet. 1993;341(8837):72-75. https://doi. org/10.1016/0140-6736(93)92555-8.

4. Compston JE, McClung MR, Leslie WD. Osteoporosis. Lancet. 2019;393(10169):364-76. https://doi.org/10.1016/S0140-6736(18)32112-3.

5. Guzon-IIlescas O, Perez Fernandez E, Crespí Villarias N, et al. Mortality afte osteoporotic hip fracture: Incidence, trends, and associated factors. J Orthop Surg Res. 2019;14(1):1-9. https://doi.org/10.1186/s13018-019-1226-6.

6. Ramisetty NM, Pynsent PB, Abudu A. Fracture of the femoral neck, the risk of serious underlying pathology. Injury. 2005;36(5):622-26. https://doi. org/10.1016/j.injury.2004.08.034

7. Torbert JT, Lackman RD. Chapter 2 Pathological Fractures. In: Pignolo RJ, Keenan MA, Hebela NM, editors. Fractures in the elderly. Aging Medicine. Springer Science+Business Media; 2011. p. 43-54. https://doi. org/10.1007/978-1-60327-467-8.

8. Siegel RL, Miller KD, Jemal A. Cancer statistics, 2020. CA Cancer J Clin 2020;70(1):7-30. https://doi.org/10.3322/caac.21590.

9. Siegel RL, Miller KD, Jemal A. Cancer statistics, 2018. CA Cancer J Clin 2018;68(1):7-30 https://doi.org/10.3322/caac.21442.

10. Damron TA, Sim FH. Surgical treatment for metastatic disease of the pelvis and the proximal end of the femur. J Bone Jt Surg Am. 2000;49:461-70. http:// www.ncbi.nlm.nih.gov/pubmed/10829199.

11. Swanson KC, Pritchard DJ, Sim FH. Abstract. The surgical treatment of metastatic disease of the femur. J Am Acad Orthop Surg. 2000;56(3):335-39. http://www.ncbi.nlm.nih.gov/pubmed/10974383.

12. Davis JA, Rohlfing G, Sagouspe K, Brambila M. Assessing the value of routine pathologic examination of resected femoral head specimens after femoral neck fracture. J Am Acad Orthop Surg. 2019;27(14):e664-8. https://doi.org/10.5435/ jaaos-d-17-00901.

13. Fornasier VL, Battaglia DM. Finding the unexpected: Pathological examination of surgically resected femoral heads. Skeletal Radiol. 2005;34(6):321-28. https://doi.org/10.1007/s00256-004-0820-8.
14. Lin MM, Goldsmith JD, Resch SC, DeAngelis JP, Ramappa AJ. Histologic examinations of arthroplasty specimens are not cost-effective: A retrospective cohort study. Clin Orthop Relat Res. 2012;470(5):1452-60. https://doi. org/10.1007/s11999-011-2149-7.

15. Liow MHL, Agrawal $\mathrm{K}$, Anderson DW, et al. Unsuspected malignancies in routine femoral head histopathologic examination during primary total hip arthroplasty: cost-effectiveness analysis. J Arthroplasty. 2017;32(3):735-42. https://doi.org/10.1016/j.arth.2016.08.017

16. Rubin G, Krasnyansky S, Gavish I, et al. Meta-analysis of unexpected findings in routine histopathology during total joint replacement. Isr Med Assoc J. 2011;13(2):80-83.

17. National Institute of Health and Care Excellence. Hip fracture: Management. BMJ. 2011;342:d2108. https://doi.org/10.1136/bmj.d2108.

18. Moodley J, Cairncross L, Naiker T, Momberg M. Understanding pathways to breast cancer diagnosis among women in the Western Cape Province, South Africa: A qualitative study. BMJ Open. 2016;6(1):1-7. https://doi.org/10.1136/ bmjopen-2015-009905.

19. Rayne S, Schnippel K, Kruger D, Benn CA, Firnhaber C. Delay to diagnosis and breast cancer stage in an urban south african breast clinic. South African Med J. 2019;109(3):159-63. https://doi.org/10.7196/SAMJ.2019.v109i3.13283.

20. Rougraff BT, Kneisl JS, Simon MA. Skeletal metastases of unknown origin. A prospective study of a diagnostic strategy. J Bone Jt Surg. 1993;75(9):1276-81. https://doi.org/10.2106/00004623-199309000-00003.

21. Li J, Zhu H, Sun L, Xu W, Wang X. Prognostic value of site-specific metastases in lung cancer: A population based study. J Cancer. 2019;10(14):3079-86. https://doi.org/10.7150/jca.30463.

22. Takagi $\mathrm{T}$, Katagiri $\mathrm{H}, \operatorname{Kim} \mathrm{Y}$, et al. Skeletal metastasis of unknown primary origin at the initial visit: A retrospective analysis of 286 cases. PLoS One. 2015;10(6):1-18. https://doi.org/10.1371/journal.pone.0129428. 